\title{
YIELD LOCUS STUDIES OF ORIENTED POLYCARBONATE: AN ANISOTROPIC AND PRESSURE-DEPENDENT SOLID
}

\author{
Ram S. Raghava and Robert M. Caddell \\ University of Michigan, Ann Arbor, Michigan, U.S.A.
}

(Received 18 March 1974)

Summary-Uniaxial and biaxial stress states were employed so as to investigate the yield behavior of oriented polycarbonate. These experimental results are compared with a theoretical yield locus based upon a yield criterion proposed for solids that are both anisotropic and pressure dependent in regard to macroscopic yield behavior. A good correlation between theory and experiment was found.

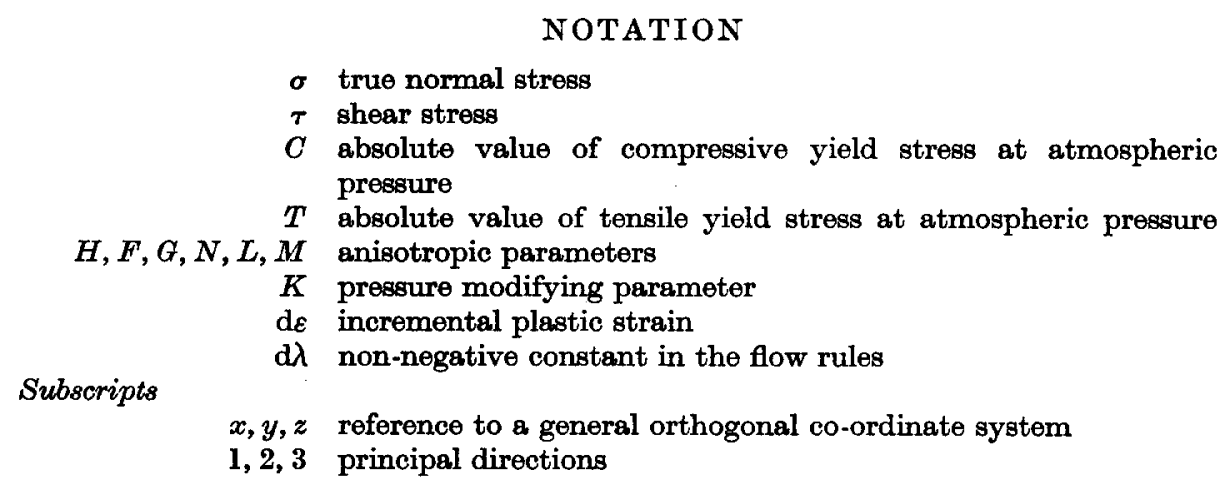

\section{INTRODUCTION}

STUDIES concerned with the macroscopic yield behavior of isotropic amorphous polymers have been discussed in some detail by Raghava ${ }^{1}$ and major findings were published by Raghava et al. ${ }^{2}$ To provide support for the yield criterion proposed by those authors, the influence of hydrostatic pressure on yielding was later discussed by Caddell et al. ${ }^{3}$ and a subsequent publication ${ }^{4}$ suggested that this same criterion was applicable to crystalline as well as amorphous polymers.

Regarding the yield behavior of anisotropic polymers, Caddell et al.5 proposed a criterion whose predictions provide an excellent correlation with experimental results reported by Rawson and Rider $^{6}$ and Shinozaki and Groves. ${ }^{7}$ Those experiments had to do with the change in tensile and compressive yield strengths of oriented polymeric sheet as a function of angular orientation in the sheet. The earlier studies of Brown et al..$^{8}$, and Rider and Hargreaves ${ }^{9}$ were also discussed in detail by Caddell et al. ${ }^{5}$

All of these studies ${ }^{5-9}$ involved the use of Hill's anisotropic yield criterion ${ }^{10}$ with pertinent modifications. Only in one $\mathrm{e}^{5}$ is it shown that plastic volume changes, as observed with polymers, are satisfied, and the use of a "Bauschinger" 
term $^{6,8,9}$ though providing improved correlation between theory and experiment, does not satisfy all basic requirements.

To investigate further the validity of a proposed yield criterion it is desirable to perform the type of studies that lead to a comparison of a theoretical yield locus and experimental findings. Such a comparison cannot be made via the type of investigations using oriented sheet materials as mentioned above. Rather, biaxial stress states must be induced; one such approach is to employ thin-walled tubes subjected to the simultaneous effects of internal pressure and axial loads.

This paper presents the findings connected with the yield locus of oriented polycarbonate and compares these experimental results with a theoretical yield locus developed from the anisotropic yield criterion suggested by Caddell et al. ${ }^{5}$

\section{ANALYTICAL BACKGROUND}

The isotropic, pressure-dependent yield criterion proposed by Raghava et al. ${ }^{2}$ is a modification of the von Mises criterion (which in its usual form is pressure insensitive). This modified form may be expressed as

$$
\left(\sigma_{x}-\sigma_{y}\right)^{2}+\left(\sigma_{y}-\sigma_{z}\right)^{2}+\left(\sigma_{z}-\sigma_{x}\right)^{2}+6\left(\tau_{x y}^{2}+\tau_{y z}^{2}+\tau_{z x}^{2}\right)+2(C-T)\left(\sigma_{x}+\sigma_{y}+\sigma_{x}\right)=2 C T .
$$

The pressure effect is introduced through the quantity $\left(\sigma_{x}+\sigma_{y}+\sigma_{z}\right)$ and $C$ and $T$ are the absolute values of compressive and tensile yield stresses messured at atmospheric pressure. Note that if $C$ and $T$ are equal, equation (1) reduces to the standard form of the von Mises criterion.

In an attempt to introduce the effects of plastic anisotropy into a yield criterion, Hill ${ }^{10}$ modified the basic von Mises criterion to the following form:

$$
H\left(\sigma_{x}-\sigma_{y}\right)^{2}+F\left(\sigma_{y}-\sigma_{z}\right)^{2}+G\left(\sigma_{z}-\sigma_{x}\right)^{2}+2 N \tau_{x y}^{2}+2 L \tau_{y z}^{2}+2 M \tau_{z x}^{2}=1
$$

where the parameters $H, F, G, N, L$ and $M$ depict the current state of anisotropy. Now equation (2) does not include any pressure effects on yielding and, in addition, assumes equivalence in the magnitude of compressive and tensile yield stresses. Since neither of these assumptions are apt to be supported where anisotropic polymers are involved, it is not surprising that the criterion expressed by equation (2) is inadequate for predictions of the yield behavior of such solids.

In a similar manner from which equation (1) was developed from the standard von Mises criterion, equation (2) may be modified to produce the following:

$H\left(\sigma_{x}-\sigma_{y}\right)^{2}+F\left(\sigma_{y}-\sigma_{z}\right)^{2}+G\left(\sigma_{z}-\sigma_{x}\right)^{2}+2 N \tau_{x y}^{2}+2 L \tau_{y z}^{2}+2 M \tau_{x x}^{2}+K_{x} \sigma_{x}+K_{y} \sigma_{y}+K_{z} \sigma_{z}=1$.

All of the parameters (both those that characterize the state of anisotropy and those that introduce the influence of pressure) are functions of the absolute values of compressive and tensile yield stresses related to the reference directions $x, y$ and $z$ which are also the principal axes of anisotropy (i.e. $C_{x}, C_{y}$ and $C_{z}$ (compression) and $T_{x}, T_{y}$ and $T_{z}$ [tension]). These parameters are defined as follows:

$$
\begin{gathered}
H+G=\frac{1}{C_{x} T_{x}}, \quad F+H=\frac{1}{C_{y} T_{y}}, \quad G+F=\frac{1}{C_{x} T_{z}}, \\
K_{x}=\frac{C_{x}-T_{x}}{C_{x} T_{x}}, \quad K_{y}=\frac{C_{y}-T_{y}}{C_{y} T_{y}}, \quad K_{z}=\frac{C_{z}-T_{z}}{C_{z} T_{z}} .
\end{gathered}
$$

For the experiments reported in this paper, which are biexial or uniaxial only, the directions $x, y$ and $z$ may be viewed as principal directions 1,2 and 3 . As a consequence, all of the terms involving shear stresses $\left(e . g . \tau_{x y}^{2}\right)$ vanish and one of the principal stresses (say $\sigma_{z}=\sigma_{3}$ ) is essumed to be zero. With these conditions equation (3) may be reduced to

$$
(H+G) \sigma_{1}^{2}+(H+F) \sigma_{2}^{2}-2 H \sigma_{1} \sigma_{2}+K_{1} \sigma_{1}+K_{2} \sigma_{2}=1 .
$$


The form expressed by equation (6) will be used exclusively in the remainder of this paper. In all biaxial cases, the stress denoted as $\sigma_{2}$ will pertain to the circumferential or hoop stress while $\sigma_{1}$ pertains to the "axial" stress. The " 1 " direction is the direction in which the orientation was induced; this is explained in detail further on.

\section{EXPERIMENTAL PROCEDURE}

\subsection{Orientation of polycarbonate rod}

Since the main goal was to conduct tube tests, it was necessary to orient a large enough bulk of material from which the tubes could be machined. This turned out to be more difficult than had been envisaged and thus it seems essentiel to document the important details. Solid bars of $2 \cdot 500$ in. $(6 \cdot 35 \mathrm{~cm})$ original diameter were machined to the dimensions as shown in Fig. 1. Initial attempts to orient the specimen by hot

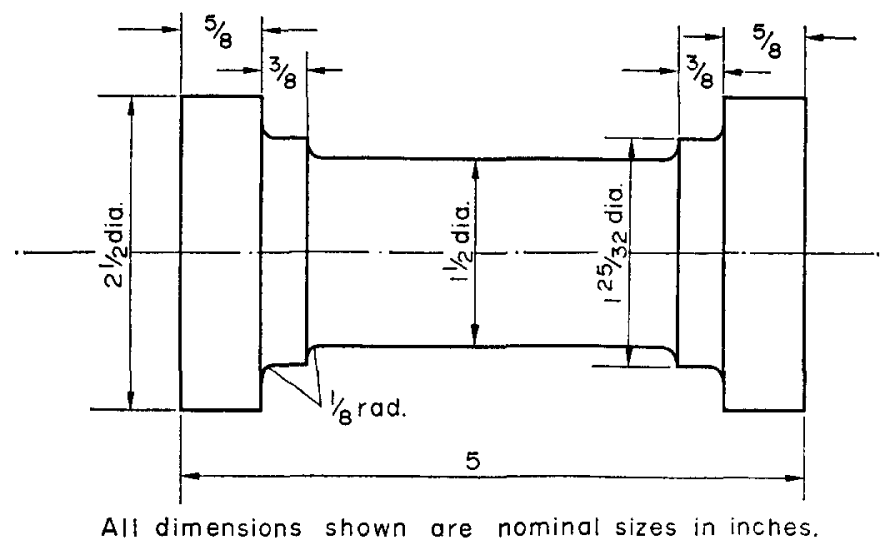

FIG. 1. Sketch showing dimensions of polycarbonate specimen prior to orientation.

stretching were not wholly successful so this approach was abandoned. Success was attained by simply subjecting the machined specimens to uniaxial tensile loading; the temperature was about $23^{\circ} \mathrm{C}$ and crosshead displacement about $0.05 \mathrm{in} / \mathrm{min}(0.13 \mathrm{~cm} / \mathrm{min})$. After the specimen necked, the neck was propagated until an oriented section of about $2.500 \mathrm{in} .(6.35 \mathrm{~cm})$ length resulted. Subsequently, a tube was machined from this specimen with the test section of the tube coinciding with the necked portion of the specimen. In this manner, all dimensional measurements obtained during subsequent testing were restricted to the highly oriented portion of the material.

Fig. 2 shows the original unoriented specimen, the specimen after adequate neck propagation and a tubular test specimen.

Several points are worth noting:

(1) After the original specimen had been machined to the dimensions shown in Fig. 1, the 1.500 dia. $(3.8 \mathrm{~cm})$ section was hand polished with 320 grit emery paper to remove all visual traces of the feed marks left by the turning tool. This was followed by a polishing action using a clear cloth buffing wheel but no buffing compound. These two steps were taken to avoid surface cracking caused by a "spreading apart" of the feed marks as tensile loads were applied. Polycarbonate seems to be very susceptible to such surface cracking unless it is finished smoothly.

(2) The technique used to produce an oriented material does not permit any great alteration in the degree or extent of orientation. Regardless of the starting diameter, the fully developed neck results from the inducement of a critical true strain as based upon area changes. In this current work, the diameter of oriented material was about $1 \cdot 140 \mathrm{in}$. $(2.9 \mathrm{~cm})$ with little variation among all specimens produced. Considering the starting diameter of 1.500 in. $(3.8 \mathrm{~cm})$ this indicates that a logarithmic or true strain of about 0.55 is induced when the stable neck has formed; in effect, this corresponds in a qualitative 
sense to the degree of orientation. If one started with a smaller or larger starting diameter, the diameter of the stable neck would be correspondingly smaller or larger but the induced strain would still be on the order of 0.55 so little would be gained if substantial variations in degree of orientation were the major goal.

(3) Relatively low crosshead speeds (i.e. "strain rate") must be used or fracture can occur before an adequate extent of neck propagation results.

(4) Three different bars of polycarbonate, purchased as commercial materials from two different suppliers, were used in this study. Both the behavior leading to orientation and subsequent measurements of key property values showed little variation; as a consequence, no concern need be expressed in regard to the possible influence of different starting structures.

\subsection{Tension tests}

The critical values of tensile yield stress required for the determination of the parameters given in equation (4) and (5) are:

(a) $T_{1}$ (or $T_{x}$ ), uniaxial tension, axial (draw) direction.

(b) $T_{2}$ (or $T_{y}$ ), circumferential or hoop tensile yield stress.

Because of size limitations it was not possible to conduct uniaxial tensile tests with specimens produced in a radial direction of the oriented material. However, as it seems quite reasonable to assume that the condition of anisotropy, as caused by orientation, was symmetric about the rod axis, such effects lead to equivalence in the radial and hoop (tangential) directions. If this is so then

$$
T_{2}=T_{3}
$$

To determine the value for $T_{1}$, specimens were produced from the oriented material, their location being shown in Fig. 3. Their cross-sections were positioned to coincide with

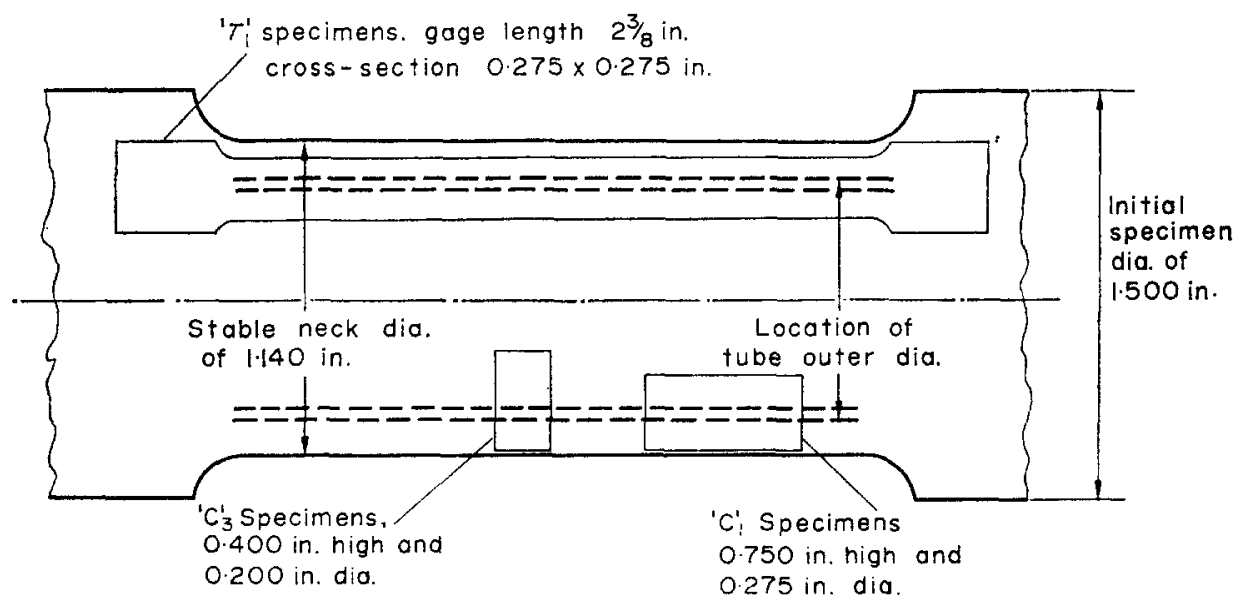

Fig. 3. Location of tensile and compressive specimens with respect to the oriented material (see Fig. 2(b) for full view of specimen).

material that formed the wall thickness of tubular specimens, and pertinent gauge section dimensions are shown. The reason for using square gauge sections is discussed later. Three tests were conducted at room temperature with this type of specimen, using an Instron machine whose crosshead speed was $0.05 \mathrm{~cm} / \mathrm{min}(0.02 \mathrm{in} / \mathrm{min})$. A standard extensometer was used to drive the recorder and a load-extension plot was obtained for each test.

One tensile test was also run using a standard tube; the location of the wall of such a tube is indicated in Fig. 3. This tube was not pressurized, rather it was subjected to an 
axial tensile load only and an extensometer adapted to the test section again drove the chart recorder. The reason for this test was to compare the stress-strain behavior with that which resulted with the three specimens just mentioned.

To determine the tensile yield stress designated as $T_{2}$ the equivalence of an open-ended tube test was conducted; two such tests were run. By using internal fluid pressure and a procedure (described in detail by Raghava ${ }^{1}$ ) that caused only a hoop stress for all practical purposes, the necessary load and dimensional changes were obtained. An extensometer noted length changes in the test section while micrometers were used to determine variations in outer diameter under increasing pressure.

\subsection{Compression tests}

In a manner somewhat like that just described, it was necessary to determine:

(a) $C_{1}$ (or $C_{x}$ ), direct compression, axial direction.

(b) $C_{3}$ (or $C_{x}$ ), direct compression, radial direction. With the assumption of rotational symmetry about the axial direction, $C_{3}=C_{2}$ (see explanation at start of Section 3.2).

The locations and dimensions of the right circular solid cylindrical specimens are shown in Fig. 3. Except for size differences all such specimens were subjected to an identical procedure, with temperature and crosshead speed being the same as used for the tensile tests. Two tests were conducted with regard to $C_{1}$ while three were completed for the $C_{3}$ measurement. A stiffness correction was obtained by running the crosshead against the load cell at the same speed used for the tests themselves. This provided a correction curve which was applied to each individual set of load/"decrease in height" data.

A single test, to evaluate $C_{1}$, was performed using a standard tubular specimen which was loaded in axial compression but not pressurized internally. Except that the extensometer was set to close up as the compressive load was applied, the procedure was similar to that in which $T_{1}$ was obtained from a tubular specimen.

\subsection{Thin-wall tube tests}

Tubes such as shown in Fig. 2(c) had a nominal test section of $2 \cdot 200 \mathrm{in} .(5 \cdot 6 \mathrm{~cm})$ length, an outer diameter of $0.890 \mathrm{in} .(2 \cdot 26 \mathrm{~cm})$ and a wall thickness of $0.040 \mathrm{in} .(0 \cdot 1 \mathrm{~cm})$. Details as to the manner in which such tubes can be produced are given by Raghava. ${ }^{1}$ In terms of a general test, a tube would be subjected to the simultaneous effects of internal pressure and axial load in such a manner that a constant stress ratio of $\sigma_{1} / \sigma_{2}$ was maintained as closely as possible; Raghava et al. ${ }^{2,4}$ describe the procedure in detail. Length changes in the test section (either extension or contraction) were determined from extensometer output to the chart drive while diametral changes (either increase or decrease) were determined from micrometer readings.

\section{RESULTS}

\subsection{Uniaxial tension and compression tests}

For each individual test discribed under Sections 3.2 and 3.3, the raw data were converted to true stress-true strain values. As will be mentioned and discussed later, volume changes during deformation of this oriented material were minor. Because of this, the concept of volume constancy was employed and the appropriate load and dimensional values were utilized to compute the stress-strain data. These were plotted on Cartesian co-ordinates and the appropriate "yield stress" derived using a 0.3 per cent offset. As explained elsewhere, ${ }^{2,4}$ this method, although as arbitrary as others, does possess the merit of consistency in defining the yield stress.* Both individual and average values for $T_{1}, C_{1}, T_{2}$ and $C_{3}$ are listed in Table 1 .

* The oriented polycarbonate used in this study did not display the type of maximum observed in the load-elongation curve for isotropic polycarbonate. Thus the use of a maximum load as the "yield load", used by a number of other investigators, cannot be used here. It is our contention, therefore, that such a way of defining yield stress is not always possible. 
Table 1. YLeld stress values for various stress Ratios

(A) Tension and compression tests

\begin{tabular}{|c|c|c|c|}
\hline & & $\begin{array}{l}\text { Individual values } \\
\text { (psi) }\end{array}$ & $\begin{array}{l}\text { Average value* } \\
\text { (psi) }\end{array}$ \\
\hline (1) & $\begin{array}{l}\text { Uniaxial tension, axial direc- } \\
\text { tion, three tests }\end{array}$ & $7400,7700,8000$ & \\
\hline & $\begin{array}{l}\text { Uniaxial tension, axial direc- } \\
\text { tion, one test with unpressur- } \\
\text { ized tube }\end{array}$ & 7700 & $T_{1}=7700$ \\
\hline (2) & $\begin{array}{l}\text { Hoop tension using open ended } \\
\text { tube, two tests }\end{array}$ & 4900,5100 & $T_{2}=T_{3}=5000$ \\
\hline (3) & $\begin{array}{l}\text { Direct compression, axial di- } \\
\text { rection, one test }\end{array}$ & -6000 & \\
\hline & $\begin{array}{l}\text { Direct compression, axial di- } \\
\text { rection, one test with unpres- } \\
\text { surized tube }\end{array}$ & -5800 & $C_{1}=|-5900|=5900$ \\
\hline (4) & $\begin{array}{l}\text { Direct compression, radial di. } \\
\text { rection, three tests }\end{array}$ & $-6400,-6550,-6650$ & $C_{2}=C_{3}=|-6550|=6550$ \\
\hline
\end{tabular}

(B) Thin-wall tube tests

\begin{tabular}{ccc}
\hline $\begin{array}{c}\sigma_{1} \\
(\mathrm{psi})\end{array}$ & $\begin{array}{c}\sigma_{2} \\
(\mathrm{psi})\end{array}$ & Stress ratio $=\sigma_{1} / \sigma_{2}$ \\
\hline 8600 & 1085 & $\mathbf{7} \cdot 93$ \\
7500 & 2400 & $3 \cdot 10$ \\
8100 & 4600 & $1 \cdot 76$ \\
6700 & $\mathbf{5 4 0 0}$ & $1 \cdot 24$ \\
3600 & 5400 & $0 \cdot 67$ \\
2700 & 5350 & $0 \cdot 50$ \\
2450 & 5320 & $0 \cdot 46$ \\
-5400 & 770 & $-7 \cdot 01$ \\
-5300 & 1180 & $-4 \cdot 49$ \\
-4420 & 1420 & $-3 \cdot 11$ \\
-3400 & 2300 & $-1 \cdot 48$ \\
-2720 & 2400 & $-1 \cdot 13$ \\
-2380 & 4130 & -0.58 \\
\hline
\end{tabular}

* These yield the following values: $H+G=0.022, H+F=0.0305, K_{1}=-0.0396$, $K_{2}=0.0473, H=G$ so $2 H=0.022$.

\subsection{Thin-wall tube tests}

In another paper ${ }^{2}$ the method for evaluating the values of $\sigma_{1}$ and $\sigma_{2}$ at the onset of yielding was discussed in detail. What is pertinent is to realize that one must define some type of strain function in order to plot stress-strain data so as to then determine the stresses at yielding. The effective strain function we have used ${ }^{2}$ is consistent with the von Mises criterion yet may be questioned as to its complete velidity here. We realize that a more correct approach would be through the use of either equivalent plastic work or equivalent plastic strain; however, with an anisotropic and pressure dependent material, the presence of the terms $K_{1}, K_{2}$ and $K_{3}$ in the flow rules presents formidable problems. Although we are currently investigating such effects, no attempt is made to include them in this current paper. For each tube test, true stress values for $\sigma_{1}$ and $\sigma_{2}$ were plotted against the effective strain function associated with the von Mises criterion; a 0.3 per cent 
offset was used for each of the two individual curves and values of $\sigma_{1}$ and $\sigma_{2}$ "at yield" were thus defined. It should be noted that the reasonableness of this approach must be supported by the ratio of $\sigma_{1} / \sigma_{2}$ (derived from the plots) which must closely agree with the known applied stress ratio for a given test; such agreement was always extremely close. All results from these tests are included in Table 1.

\section{EXPERIMENTAL RESULTS COMPARED WITH ANALYTICAL PREDICTIONS}

Using the average values for $T_{1}, C_{1}, T_{2}$ (or $T_{3}$ ) and $C_{2}$ (or $C_{3}$ ) shown in Table 1 , equations (4) and (5) were used to determine the magnitudes of $(H+G),(H+F), 2 H, K_{1}$, and $K_{2}$ for use in equation (6). These values are shown in Table 1 and when introduced into equation (6) there results,

$$
0.022 \sigma_{1}^{2}+0.0305 \sigma_{2}^{2}-0.022 \sigma_{1} \sigma_{2}-0.0396 \sigma_{1}+0.0473 \sigma_{2}=1 .
$$

For simplicity this can be altered to,

$$
\sigma_{1}^{2}+1 \cdot 39 \sigma_{2}^{2}-\sigma_{1} \sigma_{2}-1 \cdot 8 \sigma_{1}+2 \cdot 15 \sigma_{2}=45 \cdot 45
$$

By substituting numerous values of $\sigma_{1}$ or $\sigma_{2}$ into equation (10) and solving for the unknown stress, number of points resulted. These in effect, lie on the yield locus for the anisotropic material subjected to stress states in the $\sigma_{1}-\sigma_{2}$ plane; equation (10) is of course the mathematical representation of that yield locus. The solid line in Fig. 4 is the plot of

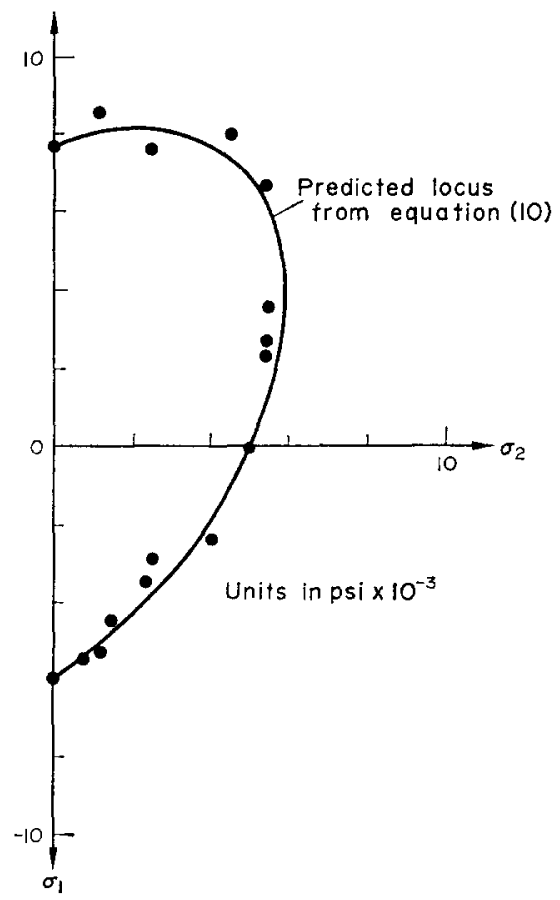

Fra. 4. Comparison between theoretical yield locus based upon the proposed criterion and actual experimental points.

equation (10) and represents the analytical predictions of yielding for the anisotropic material under consideration. The individual experimental points, all listed in Table 1, are included on that same figure; as can be seen the agreement is excellent.

For comparative purposes the full yield locus (of which that shown on Fig. 4 includes the first and fourth quadrants only) based upon equation (10) is shown in Fig. 5. Also 
included is the locus based upon Hill's criterion, given earlier as equation (2). With this latter criterion, the tensile and compressive yield strengths in a given direction are assumed equal; these magnitudes may of course vary with direction. Although in truth, $T_{1} \neq C_{1}$ and $T_{2} \neq C_{2}$ in our measurements, we have for the desired comparison, chosen the magnitudes of $T_{1}$ and $T_{2}$ for use in defining the coefficients in equation (2).

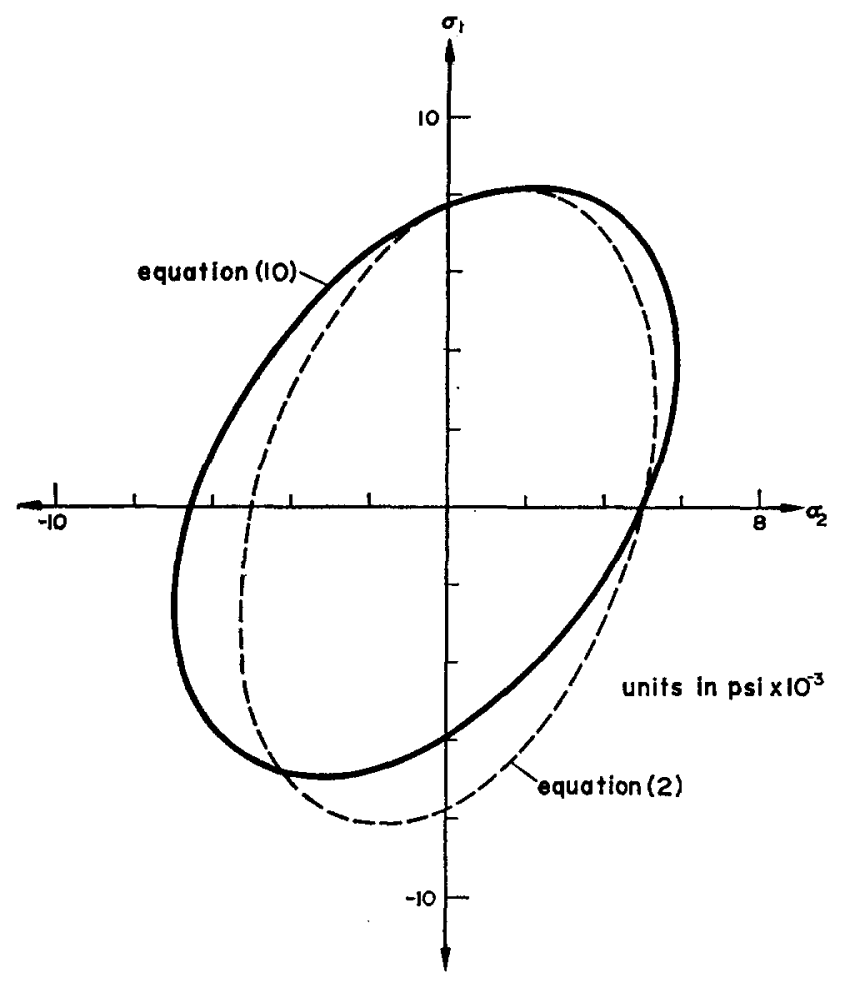

Fra. 5. Comparison of yield loci for anisotropic materials which are pressure sensitive $(-)$ and are not pressure sensitive (- . - ) .

\section{DISCUSSION}

\subsection{Anisotropic, pressure-dependent yield criterion}

As seen in Fig. 4, the agreement between theory and experiment, regarding the macroscopic yielding of oriented polycarbonate, is acceptable at the very least. Since this same criterion had previously shown excellent agreement with the tensile and compressive yield behavior of oriented sheets of polymers, ${ }^{5}$ the validity of this yield criterion is now fairly well established.

When compared with the basic. Hill criterion, as in Fig. 5, it can be seen that differences are not of equal magnitude in all four quadrants. This indicates the shortcomings of placing confidence in a particular criterion when a limited number of experiments are conducted. Certainly there are several loading paths which would indicate the onset of yielding close to the yield locus for either Hill's criterion (unmodified) or the modified criterion proposed by the authors of this paper. However, when a number of points, using loading paths in both the first and fourth quadrants, are plotted, there is little question that the correlation with the basic Hill criterion is inferior. As explained earlier, the Hill criterion, which has proved useful when the anisotropic behavior of metals* is

* Exceptions have been noted (e.g. Lee and Backofen ${ }^{11}$ and Bramley and Mellor ${ }^{12}$ ). 
involved, should not be expected to possess the desired accuracy when the materials involved are not only pressure dependent but may display different yield stresses in tension and compression.

\subsection{Strain measurements during tensile testing}

Earlier (section 3.2) it was mentioned that the tests which produced values for $T_{1}$ utilized specimens having a square gauge section. For two of those three tests, Instron Strain Sensors were adapted to the gauge section in order to messure lateral contractions as the specimen was loaded in tension; length changes were sensed by an extensometer. The output of these three pickups was amplified and fed to an $x-y-y$ recorder; loads that corresponded to particular length measurements were also recorded. From these data it was possible to determine the true stress since actual areas could be deduced. The resulting true stress-true strain data were practically identical to the plots based upon volume constancy calculations. It might also be noted that the two specimens so tested were made from material at somewhat different radial positions with regard to the cylindrical section of the oriented structure. Near equivalence of behavior implied that the structural condition due to orientation was quite uniform across the cross-section. As the sensors had been positioned to measure dimensional changes that corresponded to material which was aligned with the radial direction of the oriented section as well as at right angles to it, the two lateral strains that were computed provided a check on the assumption of rotational symmetry about the major axis of orientation.

Since the results of these two tests were almost identical, only one set of data is presented in the hope of preserving clarity. The results are shown in Fig. 6 and a few points require notice.

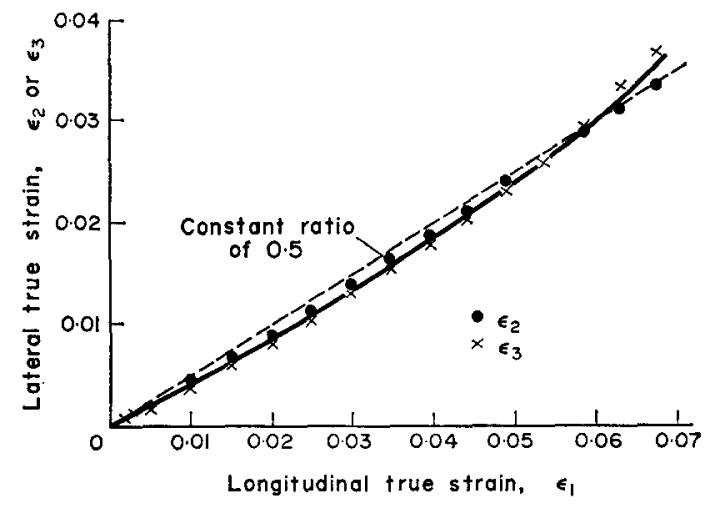

FIg. 6. Lateral strains vs longitudinal strains measured under uniaxial tension with oriented polycarbonate.

(1) Up to a longitudinal strain of 6 per cent, the two lateral strains are almost identical. The divergence that begins to appear beyond the 6 per cent value of $\varepsilon_{1}$ was probably caused by the onset of local necking in the vicinity of one of the strain sensors.

(2) The assumptions that $T_{2}=T_{3}$ and $C_{2}=C_{3}$ under Sections 3.2 and 3.3 appear to be well supported by the equivalence of $\varepsilon_{2}$ and $\varepsilon_{3}$ as seen in Fig. 6.

(3) The contraction ratio at low values of $\varepsilon_{1}(<2$ per cent) is about 0.42 ; however, at larger longitudinal strains the instantaneous value of this ratio exceeds 0.5 ; this results if one checks the slope of the solid line at a value of $\varepsilon_{1}$ in excess of 4 per cent strain. As discussed by Powers and Caddell, ${ }^{13}$ calling this Poisson's ratio can lead to disagreements so for the purposes of this paper, the phrase "contraction ratio" is used. Note that total strains are plotted in Fig. 6 and the dotted line drawn at a constant slope of 0.5 (implying constant volume) has been added for reference purposes only. 


\subsection{Comments on the flow rules}

Assuming that the plastic potential and yield function are equivalent (see e.g. Hill, ${ }^{10}$ the flow rules developed from the yield function described by equation (3) are

$$
\left.\begin{array}{rl}
\mathrm{d} \varepsilon_{x} & =2 \mathrm{~d} \lambda\left[H\left(\sigma_{x}-\sigma_{y}\right)+G\left(\sigma_{x}-\sigma_{z}\right)+\frac{1}{2} K_{x}\right], \\
\mathrm{d} \varepsilon_{y} & =2 \mathrm{~d} \lambda\left[F\left(\sigma_{y}-\sigma_{z}\right)+H\left(\sigma_{y}-\sigma_{x}\right)+\frac{1}{2} K_{y}\right], \\
\mathrm{d} \varepsilon_{z} & =2 \mathrm{~d} \lambda\left[G\left(\sigma_{z}-\sigma_{x}\right)+F\left(\sigma_{z}-\sigma_{y}\right)+\frac{1}{2} K_{z}\right] .
\end{array}\right\}
$$

With the assumption of rotational symmetry about the $x$ axis (or the 1 axis),

$$
K_{v}=K_{z} \text { and } G=H .
$$

As discussed elsewhere $\theta^{3}$ the tensile and compressive yield stresses of polymers are pressure sensitive (i.e. influenced by the mean normal stress). From equations (4) and (5) this means that the magnitudes of the various parameters are, therefore, pressure sensitive thus the flow rules as given by equation (7) are also influenced by the magnitude of the mean normal stress. An illustrative, though simplified, example will serve to illustrate this point.

During a tensile test, with the applied load in the $x$ direction, the use of the values for $H, G, K_{x}, K_{y}$ and $\sigma_{x}$ (or $T_{1}$ ) from Table 1 (all related to the use of a 0.3 per cent offset) leads to the following strain ratio from equation (7):

$$
\frac{\mathrm{d} \varepsilon_{y}}{\mathrm{~d} \varepsilon_{x}}=\frac{-H \sigma_{x}+\frac{1}{2} K_{y}}{(G+H) \sigma_{x}+\frac{1}{2} K_{x}}=-0 \cdot 408
$$

If instead of using an 0.3 per cent offset to define the various $C$ and $T$ values we had used a 1.0 per cent offset, variations in the anisotropic parameters $H, G$, etc. would result since both $C$ and $T$ would be larger. This we have done and the resulting strain ratio, as expressed by equation (8), was -0.57 . It must be admitted that total, and not plastic, strains were employed in this example so the actual numerical values of the above strain ratios are not the point of primary concern. Rather, we are merely pointing out that the flow rules for solids described by equation (7) are pressure sensitive beceuse of the $K$ terms. At this time we are pursuing a method for determining the plastic component of the total volume changes in order to investigate the suggested form of the flow rules more thoroughly.

We would add that for the criterion expressed by equation (2), the corresponding form of equation (8) reduces to

$$
\frac{\mathrm{d} \varepsilon_{y}}{\mathrm{~d} \varepsilon_{x}}=\frac{-H \sigma_{x}}{(H+G) \sigma_{x}}=-0.5 .
$$

Although $H$ and $G$ vary with the degree of plastic deformation, this ratio does not change since $H=G$ with rotational symmetry.

\section{CONCLUSIONS}

The yield locus studies, using cold drawn (oriented) polycarbonate, presented in this paper establish the validity of a proposed yield criterion as being quite suitable for predicting the macroscopic yield behavior of pressuredependent, anisotropic solids.

Although somewhat limited, experimental evidence suggests that strain ratios vary as deformation proceeds, thereby implying that the flow rules for such solids must be pressure dependent. $\dagger$

* This has been reasonably verified when the yield behavior of common metals such as low carbon steel and aluminum (which are pressure insensitive in regard to yielding) is studied.

$\dagger$ This is based upon the plastic increment of the total strain and in comparison with the flow rules used for metals, is analogous to the Levy-Mises rather than the PrandtlReuss form. 
Acknowledgements-The authors are grateful to the Horace H. Rackham School of Graduate Studies for the financial aid provided through a Faculty Research Grant. Mr. W. H. Durrant provided invaluable assistance during various phases of the experiments and Professor A. G. Atkins made several useful suggestions both during the study itself and the writing of this paper.

\section{REFERENCES}

1. R. S. Raghava, Ph.D. diss, University of Michigan, Ann Arbor, Michigan (1972).

2. R. S. Raghava, R. M. CADDELl and G. S. Y. YeH, J. Mat. Sci. 8, 225 (1973).

3. R. M. Caddell, R. S. Raghava and A. G. Atkins, Mat. Sci. Engnr. 13, 113 (1974).

4. R. S. RAghAVA and R. M. CADDELL, Int. J. mech. Sci. 15, 967 (1973).

5. R. M. Caddell, R. S. Raghava and A. G. Atkins, J. Mat. Sci. 8, 1641 (1973).

6. F. F. RAwson and J. G. RIDER, J. Polymer Sci. C, 33, 87 (1971).

7. D. Shinozaki and G. W. Groves, J. Mat. Sci. 8, 71 (1973).

8. N. Brown, R. A. DuCketT and I. M. WARd, Phil. Mag. 18, 483 (1968).

9. J. G. Rider and E. Harareaves, J. Polymer Sci. A-2, 7, 829 (1969).

10. R. HILl, Plasticity, pp. 50 and 318. Clarendon Press, Oxford (1950).

11. D. Lee and W. A. BAckofen, Trans. Met. Soc., AIME 236, 1696 (1966).

12. A. N. Bramley and P. B. Meluon, Int. J. mech. Sci. 10, 211 (1968).

13. J. Powers and R. M. CAdDELL, Poly. Eng. Sci. 12, 432 (1972). 